\title{
A multiscale direct solver for the approximation of flows in high contrast porous media
}

\author{
Akbari, Hani; Engsig-Karup, Allan Peter; Ginting, Victor; Pereira, Felipe
}

Published in:

Journal of Computational and Applied Mathematics

Link to article, DOI:

10.1016/j.cam.2019.03.028

Publication date:

2019

Document Version

Peer reviewed version

Link back to DTU Orbit

Citation (APA):

Akbari, H., Engsig-Karup, A. P., Ginting, V., \& Pereira, F. (2019). A multiscale direct solver for the approximation of flows in high contrast porous media. Journal of Computational and Applied Mathematics, 359, 88-101. https://doi.org/10.1016/j.cam.2019.03.028

\section{General rights}

Copyright and moral rights for the publications made accessible in the public portal are retained by the authors and/or other copyright owners and it is a condition of accessing publications that users recognise and abide by the legal requirements associated with these rights.

- Users may download and print one copy of any publication from the public portal for the purpose of private study or research.

- You may not further distribute the material or use it for any profit-making activity or commercial gain

- You may freely distribute the URL identifying the publication in the public portal 


\section{Accepted Manuscript}

A multiscale direct solver for the approximation of flows in high contrast porous media

Hani Akbari, Allan P. Engsig-Karup, Victor Ginting, Felipe Pereira

PII:

S0377-0427(19)30153-0

DOI:

https://doi.org/10.1016/j.cam.2019.03.028

Reference: $\quad$ CAM 12199

To appear in: Journal of Computational and Applied

Mathematics

Received date: 31 July 2018

Revised date: 11 March 2019

Please cite this article as: H. Akbari, A.P. Engsig-Karup, V. Ginting et al., A multiscale direct solver for the approximation of flows in high contrast porous media, Journal of Computational and Applied Mathematics (2019), https://doi.org/10.1016/j.cam.2019.03.028

This is a PDF file of an unedited manuscript that has been accepted for publication. As a service to our customers we are providing this early version of the manuscript. The manuscript will undergo copyediting, typesetting, and review of the resulting proof before it is published in its final form. Please note that during the production process errors may be discovered which could affect the content, and all legal disclaimers that apply to the journal pertain. 


\title{
A Multiscale Direct Solver for the Approximation of Hiows in High Contrast Porous Media
}

\author{
Hani Akbari ${ }^{1, *}$, Allan P. Engsig-Karup², Victor Gintins ₹ , Felipe Pereira ${ }^{4}$
}

${ }^{1}$ Department of Infrastructure Engineering, The University of Melhour . Parkville, Australia

* Corresponding author. hani.akbari@unime s.edu.an

2 Department of Applied Mathematics and Computer scienre, Center for Energy

Resources Engineering (CERE), Technical University of De. ${ }{ }_{\text {}}$ rk, $?$. ongens Lyngby, Denmark apek@dtu.dk

${ }^{3}$ Department of Mathematics and Statistics, The Urivers $r r$. Wyoming, Laramie, WY, USA vginting@uwyo.edu

${ }^{4}$ Department of Mathematical Sciences, The Unirorsity or Texas at Dallas, Richardson, Texas, USA luisfelipe.pereiru $\partial u^{\prime}$ lallas.edu

$$
\therefore \text { atr ct }
$$

We consider a non-overlapping domain dec umposition approach to approximate the solution of elliptic boundary value pror w... with high contrast in their coefficients. We propose a method such that initially loc: 1 solutic ns subject to Robin boundary conditions in each primal subdomain are constructed, ith (' ocally conservative) finite element or finite volume methods. Then, a novel appr ach is in roduced to obtain a (discontinuous) global solution in terms of linear combinati $~ o^{f}$ the ocal subdomain solutions. In the proposed algorithm the computation of local sc ation $f_{n}$ unions of subdomains are localized at nearest-neighbor subdomain boundaries, $t^{\prime}$, avoiding the solution of global interface problems. We remove discontinuities in a smouthing $\sim p$ that is defined on a staggered grid or dual subdomains. The resulting algorith $11 \mathrm{~L}$. naturally parallelizable and can be employed as a parallel direct solver, offering great notr atial for the numerical solution of large problems. In fact, subdomains can be consi dereu mall enough to fit well in GPUs and the proposed procedure can handle adaptive ( $\mathrm{n} \mathrm{s}$ ace) simulations effectively. Numerical simulations are presented and discussed. We a n astr ie the effectiveness of the proposed approach with two and three dimensional $\mathrm{h}^{\text {; }}$, con co st and channelized coefficients, that lead to challenging approximation problem . The . sw procedure, although designed for parallel processing, is also of value for serial calc lation .

Key w rds. E. iptic equations; Combination algorithm; Multiscale method; Robin boundary cona 'ion; F trallel computing; High contrast media. 


\section{Introduction}

Despite great advances in hardware and software, some boundary value $\mathrm{r}$ oblems arising in important applications are still computationally challenging, especially in the it rh-resolution approximation of large problems. For example, reservoir simulation inv' $\mathrm{tVt}$ large-scale timedependent coupled systems of partial differential equations with heterog ${ }^{~}{ }^{\circ} \mathrm{o} i \mathrm{~s}$, anisotropic permeability fields, horizontal wells, fractures, and so on. There are some $\mathrm{v} \mathrm{v}^{\mathrm{v}} \mathrm{la}_{\mathrm{c}_{c}} \mathrm{e}$ oil reservoirs of interest to the industry, such as those in Brazil's pre-salt layer, that $r$. 'ire . few billion cells in their discretization so that acceptable accuracy in numerical pred; tio so in obtained. Classical solution methods based on global discretizations and iterative scheme that are built in reservoir simulators can not handle problems of this magnitude. The nur erical solution of problems with these features has motivated the development of several mult : cale (] IS) and multilevel techniques $[1,2,23,24,25,28]$. For a review of a some earlier d -elop_... nts of multiscale methods we refer the reader to [11].

In MS methods, the global fine-scale problem is deromposer' into several local problems, called local solutions or multiscale basis functions, that are nu nerical solutions of local boundary value problems. They are employed to construct acc ate cc urse-scale quantities. Therefore, the goal of MS methods is to have data exchange betw nen wee and coarse quantities in order to improve the accuracy of upscaled coarse grid calculatinn this strategy is useful in applications such as uncertainty quantification, where thousands c ${ }^{c}$ simulations have to be performed [5]. For a wide range of applications these methodolog hore peen proven to be effective in terms of accuracy and improved efficiency. However, it hai $r$ sen shown that they suffer, in the context of reservoir simulation, in the presence of high v vtra ${ }^{t}$ s media, e.g. channelized fields, or highly anisotropic problems [11].

The Multiscale Finite Volume (MSF v' nucuod along with its algebraic formulation are widely used schemes in the MS framework to overcome the above-mentioned challenges $[20,22$, 26, 27]. In these procedures local solu ${ }^{+}$vin are defined in terms of Neumann boundary condition. The MSFV is based on a localizat: $n$ assu uption that ignores relations between edge cells of subdomains. No matter how man basi $f_{r}$ actions are considered, the MSFV does not converge to the fine grid solution. Howe er, $i$ has shown to be of value as an accelerator for iterative linear solvers or, in more detail as nre jonditioning matrix for iterative solvers such as GMRES or Defect Correction schemer i3).

For non-overlapping muiciscalc methods we mention initially, among others, two well-known schemes. The first one is t' $\mathrm{e} \perp+$ 'ultiscale Mortar Mixed Finite Element Method [4] that constructs local solutions with Diri, 'le $\mathrm{e}^{+}$boundary conditions. The resulting velocity fields produced with this method show lack of acc acy (discontinuous normal component of velocity at subdomain boundaries), althou\& $1 \mathrm{p}$ st r rocessing step improves such fields $[18,19]$. The second one is the Multiscale Mixed $t_{1}, h_{r} 1$ method that constructs local solutions with Neumann boundary conditions. In thi, case, the accuracy issue that arises is related to the discontinuity of pressure $[17,16]$. A dom in der mmposition based on the Robin boundary condition was considered in [7] and its ...ultisuate counterpart was developed initially in [10] for mixed finite element methods. Mc re rece tly, the Multiscale Robin Coupled Method was introduced in [9] where it was establishe' the the Mortar and Mixed Hybrid methods are extreme cases of a family of multiscale un ' nds, when parametrized by the coefficient in Robin condition of local solutions. We remark ${ }^{2}$ at the Robin boundary condition determines the relative importance of Dirichlet 
or Neumann boundary conditions in the coupling of subdomains. This point has been carefully investigated in [9].

In order to introduce the proposed algorithm, we adopt the framewor ${ }^{1}$ the Multiscale Mixed Method (see [10]), that can be viewed as one of the methods considere in [9] with constant-by-parts interface spaces. Thus, we employ Robin boundary $\mathrm{c}$, nd ions to construct local solutions on primal subdomains. The new algorithm, called recu ${ }_{\mathrm{L}}{ }^{\mathrm{V}}{ }_{\mathrm{V}}$ (or, combination) algorithm, provides local solutions on domains defined as the union of se ne or the subdomains. In our method such solutions are obtained without recalculating s su ions of boundary value problems on the larger subdomains by solving small linear syster ; ( $t$ '.e cuntinuity systems in $(21))$. This is a promising strategy for adaptive simulation [13] wh a relevant variability of coefficients of the governing equations occur only in small part, of th domain. In this class of problems, local solutions of those parts of the domain must b upda ed, while the remaining parts of the domain will be left intact, allowing for reduct: un in cumputational time. Primal subdomains can be selected small enough so that we can tal ac vantage of GPUs (Graphics Processing Units) that are much faster than CPUs. I' nwever we do not address here the implementation of the algorithm in multi-core or GPU devı 's, that we intend to consider in the near future. Our focus is in the presentation of $\mathrm{s}_{\text {- }}$ new recursive algorithm, and in the study of its approximation properties through the sor ${ }^{-i}$ ion of some challenging problems. We remark that the technique introduced here is also $r$, npplicable to other MS methods, such as $[4,9]$. Also, the proposed algorithm can be viewte as a static condensation procedure [14], which is well known in structural engineering.

Besides the multiscale mixed methods discusst ${ }^{3}$ above there are other lines of work that also aim at approximating equations with high- $\left({ }_{L}\right.$ 'as ${ }^{\prime}$ coefficients. We mention, among others, the Generalized Multiscale Finite Element Meı'od (GMsFEM). This framework was initially developed by [8]. In this method the constr ${ }^{\text {thion }}$ of enriched coarse spaces requires the solution of local eigenvalue problems that may be computationally expensive. We also mention multiscale methods that have been developed $\mathrm{fr}_{\mathrm{f}} \mathrm{Ch}_{\mathrm{a}}{ }^{1}$ enging time dependent problems, possibly involving fractures $[6,21,12]$.

The paper is organized as follo ss. In i tion 2 we introduce a model boundary value problem, which is followed by definition if lo al solutions and a description of the strategy to combine them for two nearest-neighbor ng $s$ h $d$ smains. Next, in section 3, we generalize the algorithm to an arbitrary number of $\mathrm{s}^{\prime}{ }^{\mathrm{J}} \mathrm{r}$ omains. In section 4 we show how to improve efficiency of the algorithm by balancing the accura y to a satisfactory level. As proof of concept we test the proposed schemes on sevr ial ligh contrast and channelized fields in section 5. Our conclusions are presented in section $u$.

\section{Two subdor- tirs}

Let $\Omega$ be a bour led do. 1ain in $\mathbb{R}^{n}, n=2,3$, with Lipschitz boundary $\Gamma=\partial \Omega$. The vector function $\mathbf{u}$ and sca. $r$ fr. action $P$ are related in the form

$$
\mathbf{u}=-\kappa(\mathbf{x}) \nabla P, \quad \text { in } \Omega,
$$

where $\kappa$ is a $D_{;} \ldots$. netric positive definite tensor and determines the high contrast setting of the problem. Eq ation (1) is called Darcy's equation that relates the pressure $P$ and the velocity 
u. Considering a source term $f$, define the partial differential equation

$$
L(\mathbf{u}, P)=f
$$

where a typical choice for $L$ would be

$$
L(\mathbf{u}, P)=\nabla \cdot \mathbf{u}+\mu(\mathbf{x}) P
$$

for a given a non-negative function $\mu$.

Let $\boldsymbol{\nu}$ be the outward unit normal vector on $\Gamma$. By denoting $=\mathbf{u} \cdot \boldsymbol{\nu}$ different boundary conditions on $\Gamma$ can be considered. For example, Dirichlet $\left(P=\sim{ }_{\supset}\right.$ on $\left.{ }{ }_{\supset}\right)$, Neumann $\left(u=q_{N}\right.$ on $\left.\Gamma_{N}\right)$ or Robin $\left(-\beta u+P=q_{R}\right.$ on $\left.\Gamma_{R}\right)$ boundary conditions an be et. We summarize these possibilities in the form

$$
B(\mathbf{u}, P)=q, \quad \text { on } \Gamma .
$$

We approximate numerically the solution of the boundarv value r roblem consisting of $(2)$ and (4) with a combination of (locally conservative) finite eleme t/volume methods and multiscale techniques.

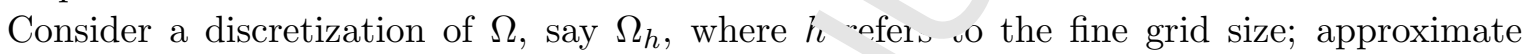
operators in (2) and (4) by finite element/volume meth. Is and look for a solution $\left\{\mathbf{u}_{h}, P_{h}\right\}$ governed by

$$
\begin{aligned}
& L_{h}\left(\mathbf{u}_{h}, P_{h}\right)={ }^{c}{ }^{c} \text {. } \quad \text { in } \Omega_{h}, \\
& B_{h}\left(\mathbf{u}_{h}, P_{h},-q_{h} \text {, on } \Gamma_{h}\right. \text {. }
\end{aligned}
$$

Under reasonable restrictions, (5) has a _.' $\mu \equiv 0$ in (3)) the solution is unique up to a con ${ }^{+}$ant. If we rewrite Equations (5) for an element of $\Omega_{h}$, say $c$, and $f_{h_{c}}$ and $q_{h_{c}}$ denote ${ }^{+} \quad$ restriction of $f_{h}$ and $q_{h}$ on $c$ and $\partial c \cap \Gamma_{h}$, respectively then

$$
\begin{aligned}
& J_{h}\left(\mathbf{u}_{i}, P_{h}\right)=f_{h_{c}}, \quad \text { in } c, \\
& \left.L_{,}, \mathbf{d}_{h}, h\right)=q_{h_{c}}, \quad \text { on } \partial c \cap \Gamma_{h} .
\end{aligned}
$$

Moreover, if $c^{\prime}$ is a nearest- ely: hor element of $c$ that shares the interface $\gamma_{c c^{\prime}}$, then $\left\{\mathbf{u}_{h}, P_{h}\right\}$ must also satisfy

$$
P=J_{c^{\prime}} \text { on } \gamma_{c c^{\prime}} \text {, and } u_{c}+u_{c^{\prime}}=0 \text { on } \gamma_{c c^{\prime}},
$$

to be considered a cor innou solution (the pair $\left\{u_{c}, P_{c}\right\}$ stands for edge values of the normal component of the $\mathrm{fl} \times \mathrm{x}$ a $\mathrm{Ad} \mathrm{r}$ essure, respectively). Consider a non-zero value on $\gamma_{c c^{\prime}}$, say $\beta$. Then, Equation (6) is ey iv lent to

$$
\mp \beta u_{c}+P_{c}= \pm \beta u_{c^{\prime}}+P_{c^{\prime}}, \quad \text { on } \gamma_{c c^{\prime}},
$$

that we call the con inuity condition.

Definition $1 L_{y}$ w continuous solution $\left\{\mathbf{u}_{h}, P_{h}\right\}$ of (5) we mean a solution that satisfies continuity cona ${ }^{\circ} i 0^{n}\left({ }^{\prime}\right)$ on all edges of elements. 
To solve (5) on smaller subdomains than the original $\Omega$ (with the hope of fitting each one of them in GPUs), consider a simple partition $\Omega_{h}=\hat{\Omega} \cup \tilde{\Omega}$ such as the one illy ,trated in Figure 1.a. Henceforth we remove the subscript $h$ for subdomains, and we refer $u$ them as primal subdomains. Later, in the discussion of the smoothing step, we will consider dua subdomains as well. The common boundary of $\hat{\Omega}$ and $\tilde{\Omega}$, call it $\gamma$, includes some inte' act; (element edges), $\gamma=\bigcup \gamma_{j}$. Thus, each $\gamma_{j}$ is a common interface edge between an elemer. $\gamma^{\prime} \tilde{\Omega}$ and an element of $\hat{\Omega}$ (a typical $\gamma_{c c^{\prime}}$ in $(7)$ ). In $\tilde{\Omega}$ and corresponding to each $\gamma_{j} \in \gamma$, we nlve e boundary value problem (see [10]):

$$
\begin{aligned}
L_{h}\left(\tilde{\mathbf{u}}_{\mathbf{j}}, \tilde{P}_{j}\right)=0, & \text { in } \tilde{\Omega}, \\
B_{h}\left(\tilde{\mathbf{u}}_{\mathbf{j}}, \tilde{P}_{j}\right)=0, & \text { on } \tilde{\Gamma}_{o}=\partial \tilde{\Omega} \cap \Gamma, \\
-\beta \tilde{u}_{j}+\tilde{P}_{j}=g, & \text { on } \gamma,
\end{aligned}
$$

where $\beta$ takes a fixed, non zero value on each $\gamma_{j}$. For $\operatorname{sim}_{1}{ }_{1}{ }_{\text {, ty }}$ r e use the index $j$ instead of $\gamma_{j}$ and keep it if no confusion arises. $g$ is a function defin. $\gamma^{\prime}$ or, and takes non-zero values on $\gamma_{j}$. In line with [10] our choice for $g$ is

$$
g=\mathbf{1}_{\gamma_{j}}= \begin{cases}1, & \text { on } \gamma_{\jmath} \\ 0, & \boldsymbol{\eta} \gamma \backslash \gamma_{j},\end{cases}
$$

that leads to

$$
\left.\left(-\beta \tilde{u}_{l}+\tilde{P}_{l}\right)\right|_{\gamma_{j}}=\delta_{\jmath_{\imath}} \quad\left\{\begin{array}{l}
\left.\gamma_{l}=\gamma_{j}, \quad l \neq 0\right) \\
\text { ๆ } l=0 \text { or } l \neq j .
\end{array}\right.
$$

In addition to $\left\{\tilde{\mathbf{u}}_{j}, \tilde{P}_{j}\right\}_{j \in \gamma}$ we compute $\left\{\tilde{\mathbf{u}}_{\mathbf{0}},{ }^{\eta_{\urcorner}}\right\}$which is the solution of

$$
\begin{aligned}
& L_{h}\left(\tilde{\sim}_{\mathbf{0}}, \tilde{\sim}\right)=\left.f_{h}\right|_{\tilde{\Omega}}, \quad \text { in } \tilde{\Omega}, \\
& B_{h} \text { ( ข }, \tilde{P}_{0}=\left.q_{h}\right|_{\Gamma_{o}}, \quad \text { on } \tilde{\Gamma}_{o} \text {, } \\
& -\beta \gamma_{0}+P_{0}=0, \quad \text { on } \gamma \text {. }
\end{aligned}
$$

Local solutions (or local basi funct s) are defined by (8) and (11). These boundary value problems can be solved by e ihe. (locally conservative) finite element or finite volume methods. Then, these solutions are $\sim \eta$ tinuous solutions within $\tilde{\Omega}$, as well as any linear combination of them. Define

$$
\begin{aligned}
& \tilde{P}=\tilde{P}_{0}+\sum_{j \in \gamma} \tilde{\alpha}_{j} \tilde{P}_{j}, \\
& \tilde{\mathbf{u}}=\tilde{\mathbf{u}}_{\mathbf{0}}+\sum_{j \in \gamma} \tilde{\alpha}_{j} \tilde{\mathbf{u}}_{\mathbf{j}},
\end{aligned}
$$

where $\tilde{\alpha}_{j}$ are unkn -n calars, and will be determined later. Extend trivially both $\tilde{P}$ and $\tilde{\mathbf{u}}$ to $\Omega_{h}$. The abo' e cons 'ruction for $\hat{\Omega}$ along with the zero extension gives

$$
\hat{P}=\hat{P}_{0}+\sum_{j \in \gamma} \hat{\alpha}_{j} \hat{P}_{j}
$$




$$
\hat{\mathbf{u}}=\hat{\mathbf{u}}_{\mathbf{0}}+\sum_{j \in \gamma} \hat{\alpha}_{j} \hat{\mathbf{u}}_{\mathbf{j}}
$$

is defined in $\Omega_{h}$, but is a continuous solution only in $\hat{\Omega}$. Note that Equation, ( $\varnothing$, $\urcorner$ nd (11) for $\hat{\Omega}$ are independent of $\tilde{\Omega}$ and can be solved in parallel (possibly in GPUs, if $\hat{\Omega}$ : sufficiently small). Now we can see that $\bar{P}:=\tilde{P}+\hat{P}, \overline{\mathbf{u}}:=\tilde{\mathbf{u}}+\hat{\mathbf{u}}$ satisfies

$$
\begin{aligned}
& L_{h}(\overline{\mathbf{u}}, \bar{P})=f_{h}, \quad \text { in } \Omega_{h}, \\
& B_{h}(\overline{\mathbf{u}}, \bar{P})=q_{h}, \quad \text { on } \Gamma_{h},
\end{aligned}
$$

where $\{\overline{\mathbf{u}}, \bar{P}\}$ is a continuous solution on $\Omega_{h}$ except on $\gamma$. Now, wa whi 'xplain how to make a $\{\overline{\mathbf{u}}, \bar{P}\}$ a continuous solution on $\Omega_{h}$ (or, on each $\gamma_{j} \in \gamma$, that $\mathrm{w}$ : refer $\rho$ as a fine grid interface edge) with an appropriate choice of the coefficients $\left\{\hat{\alpha}_{j}\right\}$ and $\left\{\alpha_{\jmath}\right\}$. In ine with $(7),\{\tilde{\mathbf{u}}, \tilde{P}\}$ and $\{\hat{\mathbf{u}}, \hat{P}\}$ must satisfy

$$
\mp \beta \tilde{u}+\tilde{P}= \pm \beta \hat{u}+\hat{P}, \quad \text { on eac } \cup_{j} \in \gamma^{\prime} .
$$

Substituting (12), (13) and (14), (15) into (17), taking (10) ' to account and by defining

$$
\theta_{l j}=\beta u_{l}+\left.P_{l}\right|_{\gamma_{j}}=\Sigma \beta u_{l}, \delta_{j},
$$

we obtain

$$
\begin{aligned}
& \tilde{\alpha}_{l}=\hat{\theta}_{0 l}+\sum_{i \in \gamma} \gamma_{j \hat{u}_{l}}, \quad l \in \gamma, \\
& \hat{\alpha}_{l}=\tilde{\theta}_{0 l}+\sum_{i \sim \nu} u_{j} \tilde{q}_{l j}, \quad l \in \gamma,
\end{aligned}
$$

or, in matrix form

$$
\left(\begin{array}{cc}
I & -\hat{i} \\
-i & I
\end{array}\right)\left(\begin{array}{l}
\tilde{\alpha} \\
\hat{\alpha}
\end{array}\right)=\left(\begin{array}{c}
\hat{\theta}_{0} \\
\tilde{\theta}_{0}
\end{array}\right)
$$

that we call the continuity syste $\iota$. The matrix entries $\hat{\theta}$ and $\tilde{\theta}$ and vectors $\hat{\theta}_{0}$ and $\tilde{\theta}_{0}$ are given in (18), hence we only need $\mathbf{u} \boldsymbol{c}_{\text {. }}$, to construct the linear system (21), and the values of $P$ on $\gamma$ play the role of auxiliar variabıs. By solving the linear system (21) we obtain $\left\{\tilde{\alpha}_{j}\right\}$ and $\left\{\hat{\alpha}_{j}\right\}$ such that continuity ce ndu. $\eta(7)$ is satisfied on $\gamma$. Thus, $\{\bar{P}, \overline{\mathbf{u}}\}$ is the desired continuous solution of (5).

Remark 2 In general th inear system (21) has a unique solution since (5) has a unique solution. In the casf of an approximation of (16)-(17) by the lowest order Raviart-Thomas spaces for pressure a. 1 - eloc iy its well-posedness has been established [9]. For a pure Neumann problem (with $\mu \equiv$ - in (v, the solution is unique up to a constant, and therefore (21) has one degree of freedom

\section{More than two subdomains}

Next, we ' ons ucs multiple subdomains (with many elements) that makes an effective use of multi-core pa allel machines. Thus, in line with Figure 1.b, consider a partition of $\tilde{\Omega}$, say $\tilde{\Omega}_{a} \cup \tilde{\Omega}_{b}$, 
(a)

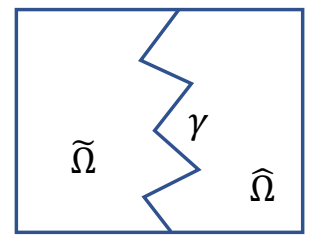

(b)

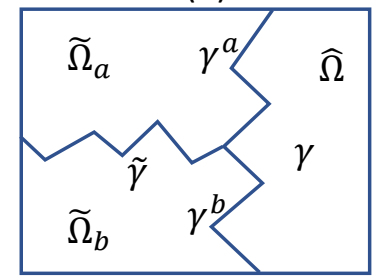

(c)

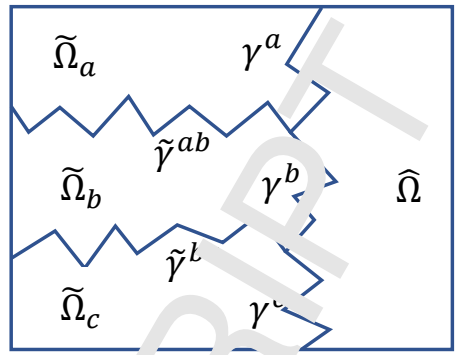

Figure 1: Partition of $\Omega_{h}$. (a) $\Omega_{h}=\tilde{\Omega} \cup \hat{\Omega} \quad$ (b) $\tilde{\Omega}=\tilde{\Omega}_{a} \cup \tilde{\Omega}_{b} \quad$ (c) $\tilde{\Omega}=\tilde{\Omega}_{a} \cup \tilde{\Omega}_{b} \cup \tilde{\Omega}_{c}$.

their common boundary, $\tilde{\gamma}$, and $\gamma^{a}$ and $\gamma^{b}$ that correspon !, $\mathrm{r}^{\mathrm{r}} \mathrm{s} \in$ tively, to their intersections with $\gamma$ :

$$
\tilde{\gamma}=\partial \tilde{\Omega}_{a} \cap \partial \tilde{\Omega}_{b}, \quad \gamma^{a}=\partial \tilde{\Omega}_{a} \cap \gamma, \quad \gamma^{b}=\partial \Omega_{b}, \gamma, \quad \gamma=\gamma^{a} \cup \gamma^{b} .
$$

For each $\gamma_{j}^{a} \in \tilde{\gamma} \cup \gamma^{a}$ and $\gamma_{j}^{b} \in \tilde{\gamma} \cup \gamma^{b}$ we solve (in par^llel, '^r . and (11) defined on smaller subdomains $\tilde{\Omega}_{a}$ and $\tilde{\Omega}_{b}$. We ${ }_{\llcorner}$' 'd to address the question of how to represent a local solution of $\tilde{\Omega}$, say $\tilde{P}_{j}$ for $j \in \gamma^{a} \subset$ " in terms of $\left\{P_{a_{j}}\right\}$ where $a_{j} \in \gamma^{a} \cup \tilde{\gamma}$ and $\left\{P_{b_{j}}\right\}$ where $b_{j} \in \gamma^{b} \cup \tilde{\gamma}$. For simplicity in the discussıun below we refer only to the pressure variables, and the corresponding equations for , he dux variables (such as Equation (15) that corresponds to Equation (14)) are omitted. "No she v that

$$
\tilde{P}_{j}=\tilde{P}_{a}+\tilde{P}_{b}, \text { where } \tilde{P}_{a}=\tilde{\sim}_{\tau_{i}}: \sum_{k \in \tilde{\gamma}}^{-} \tilde{\alpha}_{a_{k}} \tilde{P}_{a_{k}}, \text { and } \tilde{P}_{b}=\sum_{k \in \tilde{\gamma}} \tilde{\alpha}_{b_{k}} \tilde{P}_{b_{k}} .
$$

It is clear that $\tilde{P}_{j}$ satisfies Equatior (8) a. d we need to address its continuity over $\tilde{\gamma}$. In line with Equation (21), the solution of $\iota_{\text {. }}$ - con inuity system

$$
\left(\begin{array}{cc}
I & -\tilde{c}_{b} \\
-\theta_{a} & I
\end{array}\right)\left(\begin{array}{c}
\tilde{\alpha}_{a} \\
\tilde{\alpha}_{b}
\end{array}\right)=\left(\begin{array}{c}
0 \\
\tilde{\theta}_{a_{j}}
\end{array}\right)
$$

gives the coefficients $\tilde{\alpha}_{a}=\left\{\tilde{\alpha}_{a_{j}}\right\}$ and $\tilde{\alpha}_{b}=\left\{\tilde{\alpha}_{b_{j}}\right\}$ such that $\tilde{P}_{j}$ becomes continuous over $\tilde{\gamma}$ and hence in the subdomain $\iota_{a}$. As in Equation (18) the matrix entries and vectors in Equation (23) are given by

$$
\left[\left.\tilde{\theta}_{a}\right|_{l k}=\left.2 \beta u_{l}\right|_{\gamma_{l}^{a}}+\delta_{l k}, \quad\left[\tilde{\theta}_{a_{j}}\right]_{l}=\left.2 \beta u_{a_{j}}\right|_{\tilde{\gamma}_{l}^{a}} .\right.
$$

Thus, we have determı $\gamma\left\{\check{P}_{j}\right\}_{j \in \gamma}$ in terms of solutions computed in smaller subdomains. We remark that if $\mathrm{w}^{\prime}$ were olving a time dependent problem and if the changes (in time) of the coefficient $\kappa$ in ( $i$ ' were ocalized in $\tilde{\Omega}_{a}$ from a time step to the next, then it would suffice to update only tr. ocar sunctions in $\tilde{\Omega}_{a}$, and then compute $\tilde{P}_{j}$ with the new matrix entries in (23). It is easy to : ee that $\left\{\tilde{P}_{j}\right\}$ for $j \in \gamma^{b}$ and $\tilde{P}_{0}$ are given by

$$
\tilde{P}_{j}=\tilde{P}_{b_{j}}+\sum_{k \in \tilde{\gamma}} \tilde{\alpha}_{b_{k}} \tilde{P}_{b_{k}}+\sum_{k \in \tilde{\gamma}} \tilde{\alpha}_{a_{k}} \tilde{P}_{a_{k}},
$$


and

$$
\tilde{P}_{0}=\tilde{P}_{b_{0}}+\sum_{k \in \tilde{\gamma}} \tilde{\alpha}_{b_{k}} \tilde{P}_{b_{k}}+\tilde{P}_{a_{0}}+\sum_{k \in \tilde{\gamma}} \tilde{\alpha}_{a_{k}} \tilde{P}_{a_{k}}
$$

Note that coefficients in (22), (24) and (25) are not the same and, again, for simplluity, we have kept the same notation. Moreover, $\left\{P_{j}\right\}$ is the solution of a Robin bound $₫$ ry alue problem (see Equation (8)) and, hence, the coefficients are the unique solution of then - rresponding linear system, no matter if the underlying problem is a pure Neumann boundar, value problem or not.

To sum up, we have combined two subdomains $\tilde{\Omega}_{a}$ and $\tilde{\Omega}_{b}$ or, equi al ntly, we have obtained a local solution in terms of local solutions of smaller subdomains. In 'are lel numerical simulation this can happen for any pair of subdomains, or even for more than twe as we discuss next.

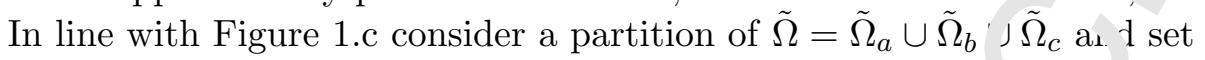

$$
\tilde{\gamma}^{a b}=\partial \tilde{\Omega}_{a} \cap \partial \tilde{\Omega}_{b}, \quad \tilde{\gamma}^{b c}=\partial \tilde{\Omega}_{b} \cap \partial \tilde{\Omega}_{c}, \quad \gamma^{z}=\partial \tilde{\Omega}_{z}, \cdots, z=a, b, c .
$$

We show that $\tilde{P}_{j}$ for $j \in \gamma^{a} \subset \gamma$ is given by

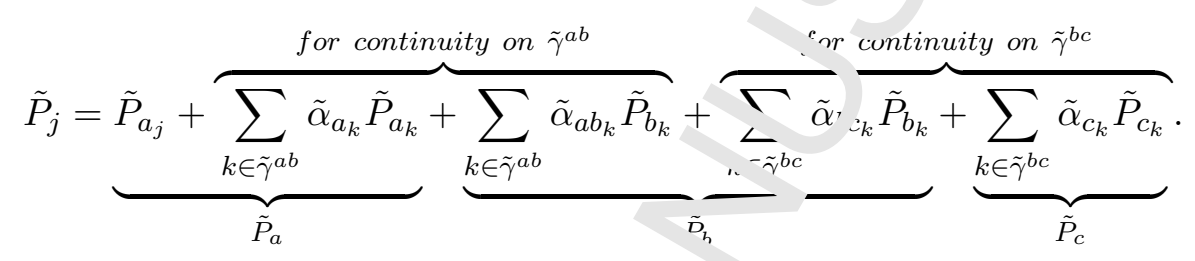

Clearly $\tilde{P}_{j}$ satisfies (8). Continuity on $\tilde{\gamma}^{a b}$ and $\cdots$ ?ires that

$$
\mp \beta \tilde{u}_{a}+\tilde{P}_{a}= \pm \beta \tilde{u}_{b}+\tilde{P}_{b} \text {, on } \tilde{\gamma}^{a b} \quad \text { nd } \mp \beta \tilde{u}_{b}+\tilde{P}_{b}= \pm \beta \tilde{u}_{c}+\tilde{P}_{c} \text {, on } \tilde{\gamma}^{b c} \text {. }
$$

Substituting terms of $(26)$ in $(27)$ gives

$$
\left(\begin{array}{cccc}
I & -\tilde{\theta}_{a b} & -\tilde{\theta}_{b c} & \vdots \\
-\tilde{\theta}_{a} & I & n & 0 \\
0 & 0 & I & -\tilde{\theta}_{c} \\
0 & -\tilde{\theta}_{j}^{\prime} & -\tilde{\vartheta}^{\prime} & I
\end{array}\right)\left(\begin{array}{c}
\tilde{\alpha}_{a} \\
\tilde{\alpha}_{a b} \\
\tilde{\alpha}_{b c} \\
\tilde{\alpha}_{c}
\end{array}\right)=\left(\begin{array}{c}
0 \\
\tilde{\theta}_{a_{j}} \\
0 \\
0
\end{array}\right)
$$

where, for example,

$$
\left[\tilde{\theta}_{a b}\right]_{l k}=\left.2 \beta u_{b_{k}}\right|_{\tilde{\gamma}_{l}^{a b}}+\delta_{l} \text { for } l, n=\tilde{\gamma}^{a b}, \quad\left[\tilde{\theta}_{a b}^{\prime}\right]_{l k}=\left.2 \beta u_{b_{k}}\right|_{\tilde{\gamma}_{l}^{b c}} \text { for } l \in \tilde{\gamma}^{b c} \text { and } k \in \tilde{\gamma}^{a b} .
$$

This procedure can be extended any number of subdomains. However, it is important to note that it is not our in en 'on to construct solutions on larger subdomains at all. We have computed local solution $>n$ the smallest subdomains and want to know what would be their coefficients in the glob 1 solut 1 , i.e., $P_{h}$ in (5), that is a linear combination of all local solutions. Thus, we now focus $\mathrm{n} \mathrm{h}$ sw $\dagger$, determine $\left.P_{h}\right|_{\tilde{\Omega}_{a}}$. For simplicity, instead of a linear combination $\sum_{k \in \gamma} \alpha_{k} P_{k}$, we intrnduc a matrix $\mathbf{P}$ such that the $P_{k}$ s are set to be its columns and we can write $\sum_{k \in \gamma} \alpha_{k} P_{k}=\mathbf{P} \alpha$. Hence,

$$
P \cdot 1_{\tilde{\Omega}_{a}}-\left.\left(-h||_{\tilde{\Omega}}\right)\right|_{\tilde{\Omega}_{a}}=\tilde{P}_{a_{0}}+\left.(\tilde{\mathbf{P}} \tilde{\alpha})\right|_{\tilde{\Omega}_{a}}=\tilde{P}_{a_{0}}+\left.\tilde{\mathbf{P}}\right|_{\tilde{\Omega}_{a}} \tilde{\alpha}=\tilde{P}_{a_{0}}+\tilde{\mathbf{P}}_{a} \mathbf{A}_{a} \tilde{\alpha}
$$

where $\tilde{\alpha}$ is th' soluti in of (21). $\tilde{P}_{a_{j}}$, that is one column of $\tilde{\mathbf{P}}_{\tilde{\Omega}_{a}}$, is computed in (28), and if we put the coefficit_.. of (28) in a matrix $\mathbf{A}_{a}$, then one can derive the last equality. Equation (29) displays tL' $\mathrm{g}$ ' swai solution in terms of local solutions $P_{a_{0}}$ and $\tilde{\mathbf{P}}_{a}$, this is the key point of our method that reeds to be carefully implemented. We summarize our findings as follows: 
Remark 3 After computing local solutions of (8) (corresponding to each fine grid interface edge) and (11) and obtaining their coefficients by solving the continuity syst $m$ (7) and (29), we get the desired continuous solution of (5). Local solutions can be comp' ${ }^{\prime}+1$ in parallel and combined as needed efficiently. The continuity system require values (such as "ux) only at subdomain boundaries, and with a careful implementation, its solution car be, andled in parallel. Thus, we have developed a parallel direct solver. We refer to it as the recu, in (or, combination) approach and denote the solution by $\left\{P_{c l}, \mathbf{u}_{c l}\right\}$, where cl refers to (lintu.') combination of local solutions.

We remark that, although we can compute $P_{h}$ (the exact, fine grid sor ${ }^{{ }^{\prime}}$ on of (5)), in applications such as simulation of porous media flows with billions of degre ss of fretlom, one may be able to use approximate solutions provided that they can be obtai ed mo e efficiently, at the cost of reduced accuracy. Next we discuss a strategy to find an anpr -in ation to $P_{h}$, that leads to improved efficiency in the computation of the recursive sol ttior. $^{\text {. }}$

\section{Efficiency}

Up to this point in the development of our method a : mily of local solutions is computed for each subdomain corresponding to each fine grid int … . . . nunent edge of each subdomain. Then, the fine grid continuous solution $P_{h}$ is obtained. Now, that even in parallel implementation on many-core devices, this might be a very expen $v_{c}$. $^{\prime}{ }^{\prime}$ lation (possibly computed in an offline stage of the computation).

Efficiency of our method can be improvec $\iota_{\nu}$ re sxing the continuity requirement. To relax this restriction, we construct less local solutic is within each subdomain, each one of them corresponding to a set of interface edges. (u- sequently, the continuity systems becomes smaller, more subdomains can be combined at once, less solutions and coefficients must be saved, and less data needs to be interchanged $\mathrm{b}$ ' w wet. processes in parallel devices.

We explain the recursive approa ${ }^{h}$ for $t$ t e partition shown in Figure 1.a and it can be easily derived for other cases. The pro edure ' scribed here is motivated by the introduction of an intermediate $\bar{H}$ length scale in [ 0 ]. Let $n \geq 1$ to be the number of interface edges employed in the construction of a local sol ،tioı. T $\mathrm{T}^{\prime}$ en, if we refer to Figure 1.a, we write $\gamma=\cup_{j} \psi_{j}$ where each $\psi_{j}$ consists of $m$ interf $~ \cdots$ edges or $\gamma_{j}$ s and we write $\psi_{j}=\cup_{i=1}^{m} \gamma_{j_{i}}$. Obviously, each $\psi_{j}$ may consist of a distinct number ${ }^{c}{ }^{c}$ interface edges, say $m_{j}$. However, for simplicity, we have considered only one valuf for $m_{j}$ to be $m$. If we consider $m=1$ then $\psi_{j}=\gamma_{j}$, then Remark 3 holds.

As before, we mu $i$ fix a ..on-zero value for $\beta$ on each $\psi_{j}$ to compute local solutions on both $\tilde{\Omega}$ and $\hat{\Omega}$. We et $\beta$ tr be a constant on $\gamma_{j}$ (see, however, [9] for other choices of this parameter). Extrems vas $\cdot o$ of $\beta$ might be of interest, as discussed also in [9]. If we set $\beta=0$ then the continu ty col lition (7) implies continuity of $P$ in (6) and the local solutions are defined in terms 6 . Diric' let boundary value problems. On the other hand, very large values for $\beta$ imply conti uliy of $u$, and if it significantly dominates $P$ then (8) becomes close to a Neumann boundary va. te prok 'em. We also set a non zero value $g$ on each $\psi_{j}$ and for simplicity we use (9). In summa. ne local solutions for $m \geq 1$ are the same as (8) and (11), except for the boundary alu ${ }^{\prime} 9$ ) and (10) where $\gamma_{j}$ is replaced by $\psi_{j}$, thus changing $\tilde{P}$ and $\hat{P}$ in (12) and (14), respeci rely: 


$$
\tilde{P}_{m}=\tilde{P}_{0}+\sum_{\psi_{j} \in \gamma} \tilde{\alpha}_{j} \tilde{P}_{j}, \quad \hat{P}_{m}=\hat{P}_{0}+\sum_{\psi_{j} \in \gamma} \hat{\alpha}_{j} \hat{P}_{j}
$$

We have introduced the subscript $m$ to indicate that we are referring $+-\psi_{j}$. A first result concerning this construction is that for $m>1$ there are no coefficients $\left\{x_{j}\right\}_{{ }_{j}}$ and $\left\{\hat{\alpha}_{j}\right\}_{\psi_{j}}$ that give the desired continuous solution $P_{h}$. In other words we can find $\bar{P}$ cu - inuous on each $\psi_{j}$ (but not on each $\gamma_{j}$ ). Thus, we get a discontinuous approximation, in con ast with case $m=1$. However, we still refer to it as $\left\{P_{c l}, \mathbf{u}_{c l}\right\}$.

Remark 4 Our goal is to find a continuous approximation of $\left\{P_{h}, \mathbf{u}_{h}{ }^{\prime}\right.$, Thus, a discontinuous approximation $\left\{P_{c l}, \mathbf{u}_{c l}\right\}$ must be refined. This is the subject of ? ost pr. cessing or smoothing step to be discussed in next subsection.

Note that different values for the coefficients $\left\{\tilde{\alpha}_{j}\right\}_{\psi_{j}}$ an $\left\{\hat{\alpha} j \psi_{\text {. }}\right.$ can be obtained. For example, one might consider minimizing the overall discontinuity on $\gamma$ Here we address continuity on each $\psi_{j}$, so in (17) we replace $\gamma_{j}$ with $\psi_{j}$. A question $\therefore$ at arises is how to compute $\theta_{l j}$ in (18). Actually, when we compute a local solution, a s $s^{1} 1$ tion c a fine grid is obtained, hence we can only compute $2 \beta u_{l}+\delta_{l j}$ for each $\gamma_{j}$, and not $f_{c} \cdot$ a $\psi_{j}$. Thus, we assign the (arithmetic) average of $2 \beta u_{l}+\delta_{l j}$ of $m$ interface edges to $\theta_{l j}$. The $r_{t}$-aining portion of the argument for combining subdomains remains unaltered for $m>$ : and $m=1$. We obtain $\left\{P_{c l}, \mathbf{u}_{c l}\right\}$, an approximation for (5), that is discontinuous at th hnindaries of the primal subdomains, and has to be post-processed to become a continuous apt $\mathrm{C}$.imation.

\subsection{Smoothing step}

Starting from $\left\{P_{c l}, \mathbf{u}_{c l}\right\}$ (discontinuous at the houndaries of primal subdomains) we describe a method (motivated by a related procenure introduced in [10]) that gives a continuous approximation to either $P_{h}$ or $\mathbf{u}_{h}$. It is $\mathrm{b}$ sed oı a primal/dual iteration, such that the boundaries of primal/dual subdomains take bou. tary values from the interior of each other. If only one iteration is considered we call it , ual smouthing solution that is denoted by $\left\{P_{d s}, \mathbf{u}_{d s}\right\}$. One can take the dual solution as the finc' ${ }^{\prime} r_{f}$, ult However, at the cost of one additional local solution in primal subdomains, the appre simatı can be refined one more time. We call it primal smoothing, denoted by $\left\{P_{p s}, \mathbf{u}_{p s}\right\}$. r hu the cost of smoothing step just described consists of two local solutions. Next we discus the eftect of both dual and primal smoothing steps in numerical experiments.

\section{$5 \quad$ Numerical ex seriments}

The recursive alg sithm described above to construct a global solution is independent of the contrast of the ur terlyin; coefficients of the governing equations. We discuss some examples to assess the imp or $\ldots$ (or, equivalently, the number $m \geq 1$ of interface edges employed in the construction of a loc solution) and the choice of boundary conditions (Dirichlet or Neumann)

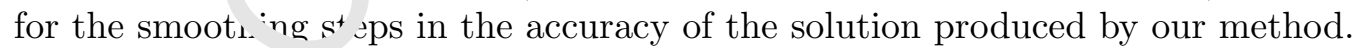

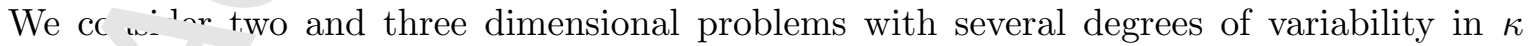
in (3). In ample $4, \mu$ is non zero, and it is set to zero in the remaining examples. The 
permeability data for all examples considered here (except for Example 4) are extracted from the SPE 10 project [15], that is one of the most challenging databases regarding the high contrast in the data. In all examples first we compute local solutions with constar, $q \equiv 1$ (for other possibilities, see [9]), $g$ as in (9), and then they are combined with our proposeu ipproach to obtain the approximation $\mathbf{u}_{c l}$. Then, we refine it with a dual followed $r_{y}$ a primal smoothing steps, to obtain $\mathbf{u}_{d s}$ and $\mathbf{u}_{p s}$. For plotting purposes we compute for t. ${ }^{-r}$ cell, say $c$, either cell-centred values for $P$ or the magnitude of the flux on the cell edge, ana hen we plot the cell-wise relative error

$$
e_{c}=\frac{\left\|\mathbf{u}-\mathbf{u}_{h}\right\|_{c}}{\left\|\mathbf{u}_{h}\right\|_{c}} .
$$

The reported relative errors refer to the $l^{2}$ norm of this functic 1 .

\subsection{Example 1}

We consider a $48 \times 48$ high contrast field $(\kappa)$ that is plotte $r$ in Figr ie 2.1. The reference solution for this example, $P_{h}$, and the magnitude of the reference $\mathbf{u}_{h} \dot{a}_{-}$' displayed in Figures 2.2 and 2.3, respectively. The boundary conditions are set as Diricis + ${ }^{+}$on t' e left and right boundaries, with values zero and one, respectively. Zero Neumann con ${ }^{i}$ tion is imposed on the top and bottom boundaries. We consider a $16 \times 16$ local grid for $n n k$ mal subdomains such that we have a partition of the domain in $3 \times 3$ primal subdoma $a_{-}$. By setting $m=8$ in the construction of local solutions and applying the recursive $a_{i} \ldots h$ we obtain a recursive solution $\mathbf{u}_{c l}$. The corresponding relative error (31) is shown in Figu" 2.4 .

We assigned the maximum error to cells $\mathrm{w}$.. val as above a threshold to highlight the largest values of errors and, as expected, larger error va, 'es are concentrated close to the green lines (the boundaries of primal subdomains). We se ${ }^{\text {the }}$ velues of $\mathbf{u}_{c l}$ along the white line as Neumann boundary conditions for dual subdomains to ontain $\mathbf{u}_{d s}$. The corresponding error (computed using (31)) is shown in Figure 2.5. \& t nected, errors around green lines have been reduced and the largest errors are concentr ted arc und white lines. However, they are much smaller than the error associated with $\mathbf{u}_{c}$. Finc ${ }^{1{ }^{\prime}{ }_{v}}$, we apply primal smoothing. It gives a satisfactory solution, $\mathbf{u}_{p s}$, that is shown in $F$ igu 2.6 . In Figure 3 the relative error for different values of $m$ s are plotted in log scale. Fc $m_{\iota}-1$, he relative error is of the order of machine precision.

\subsection{Example 2}

The $56 \times 200$ high con ${ }^{+}$ast permeability field considered in this study is plotted in Figure 4.1. Boundary conditirns at pure Neumann for all boundary faces except for the bottom left (non-zero inward $\mathrm{Ne}^{\prime} \mathrm{ma}$ ) and top right (Dirichlet) cells. We take 8 subdomains as primal subdomains with part onir $\mathrm{g}$ only in the $\mathrm{x}$ direction. We construct local solutions for $m=8$ and $m=4$ and $\mathrm{r}$ port the result in Figures 4.2 and 4.3, respectively. We set the values of $\mathbf{u}_{c l}$ and $\mathbf{u}_{d s}$ as Neum. nn bov ıdary conditions for the dual and primal smoothing steps, respectively. By looking at $r$ is a . wix with 56 rows and 200 columns, we compute the relative error in log scale for each colum,$j$,

$$
\frac{\left\|\mathbf{u}-\mathbf{u}_{h}\right\|_{C o l_{j}}}{\left\|\mathbf{u}_{h}\right\|_{C o l_{j}}}
$$


(1)

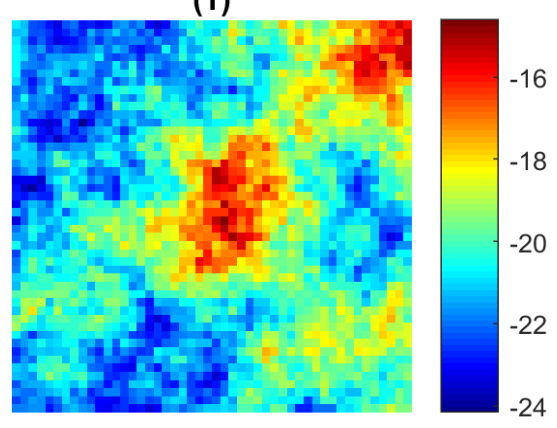

(3)
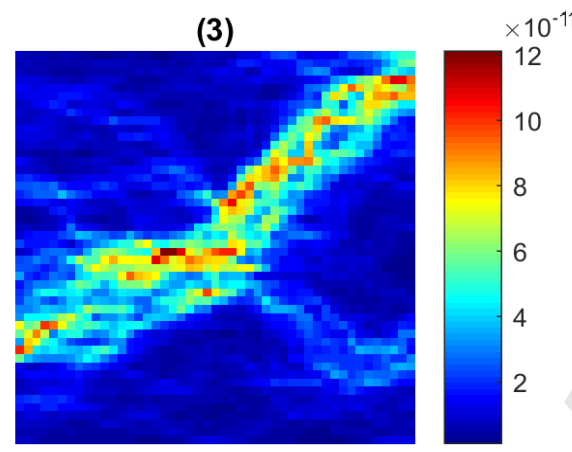

(5)
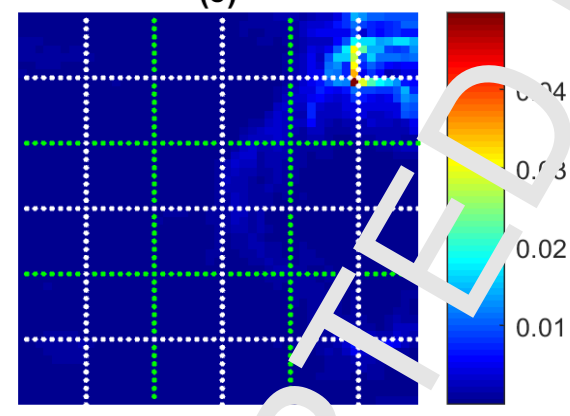

(2)

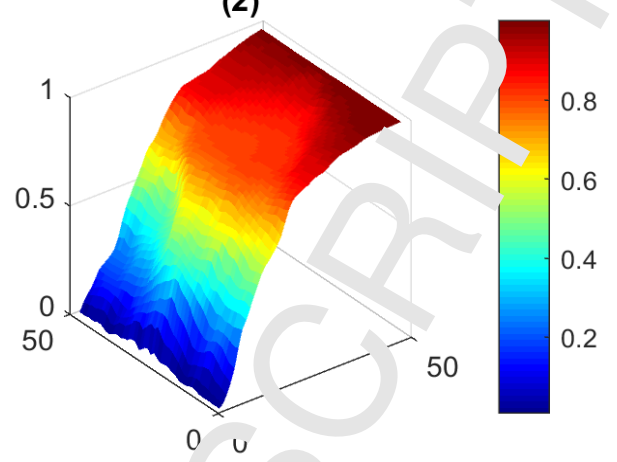

(4)
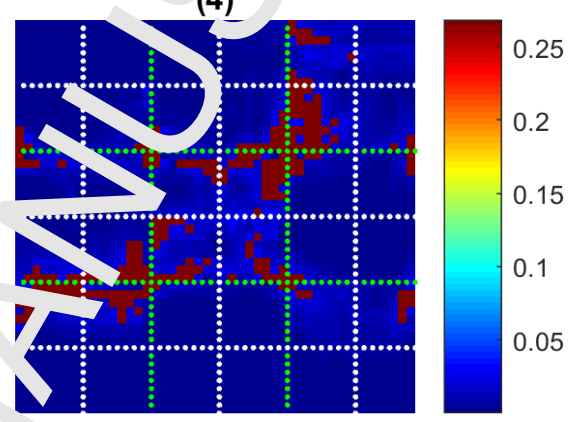

(6)
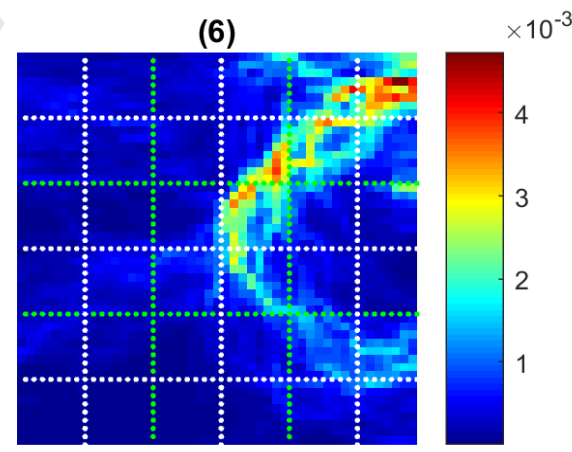

Figure 2: Example 1 A $\angle 3 \times 18$ model with Dirichlet boundary values on left and right. Primal and dual subdomains a. $\mathrm{d}^{\mathrm{d}}$ iermined with green and white lines respectively. (1) Logarithmic scale of field whir a shows nigh contrast media. (2) Reference $P_{h}$. (3) Magnitude of reference $\mathbf{u}_{h}$. (4) Error of c rmbina ion of local solutions. The largest errors are highlighted and around of boundary of pr: ial wudomains. (5) Effect of dual smoothing that removes error around primal subdomains nd int sduces small errors on boundary of dual subdomains. (6) Effect of primal smoothing. 


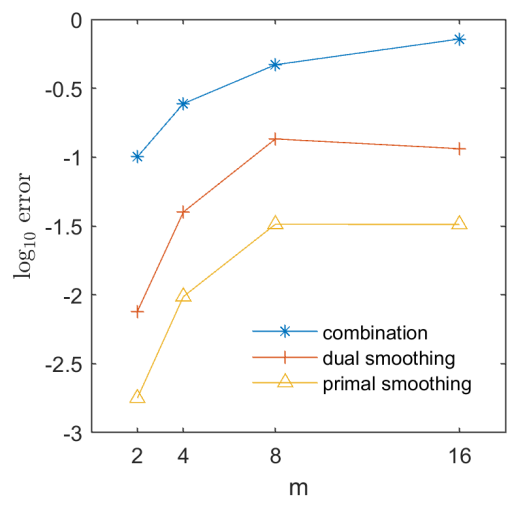

Figure 3: Example 1. Relative error in log scale for combi tation dual and primal smoothing, for $m=16,8,4,2$. For $m=1$ the relative error is of the or ar of $\mathrm{m}$ achine precision.

and plot it. Hence at each one of the 25 columns, that re the oundaries of primal subdomains (position of the discontinues), we expect a peak for nelau. - errors as can be seen with green lines in figures. However the error is smaller in the middte "egion of primal subdomains. We set these middle values as boundary conditions for dua ' subdomains, and we expect to see a peak for relative errors, shown in red curves. Primal monothıng gives the final result.

\subsection{Example 3}

We consider a $56 \times 200$ high contrast che nolizt ${ }^{1}$ field that is plotted in Figure 5.1. This type of field is very challenging for multi-scale tec niques and we show the efficiency of the proposed method in this case. Boundary conditions are set as pure Neumann for all boundary faces except for the bottom left (non-zero inwar' Neus ann) and top right (Dirichlet) cells. We consider 8 primal subdomains with partitioning $\eta \mathrm{ly}$ ir the $\mathrm{x}$ direction. Local solutions are constructed for $m=8$ and $m=4$ and we set the alves $\mathrm{O}_{1} P_{c l}$ and $P_{d s}$ as Dirichlet boundary conditions for dual and primal smoothing, respectiv ' $\mathrm{v}$. By ' Joking at $\kappa$ as a matrix with 56 rows and 200 columns, we compute the relative error $\Omega \log \cdots$ " e for each column $\mathrm{j}$ for $P$ as in (32), and plot it. Results are reported in Figures 5.2 m 5.3 for $m=8$ and $m=4$, respectively. At integer multiples of 25 columns, that are the nositions of discontinues, we expect a peak for relative errors, that are smoothed in smoothi $\mathrm{ig} \mathrm{s}$ `p. As an assessment of the accuracy of the proposed method we show, for $m=4$, the cell- $v_{v}$ e error (31) for $P_{p s}$.

\subsection{Example 4}

We consider in th s exai ple a non zero $\mu$ in (3). The domain $\Omega$ is $(0,1) \times(0,1)$ discretized by a $48 \times 48$ mesh. We set th: source term and boundary conditions such that $P=x \cos (\pi y)$ is the exact solution vi (3) ior

$$
\kappa=\left(\begin{array}{ll}
x & 0 \\
0 & y
\end{array}\right), \quad \mu=0.01 .
$$

The refert ce surutions for $P_{h}$ and the magnitude of $\mathbf{u}_{h}$ are plotted in Figures 6.1 and 6.2, respectively. The recursive solution is plotted in 6.3 and, as in Example 1, the largest values of 

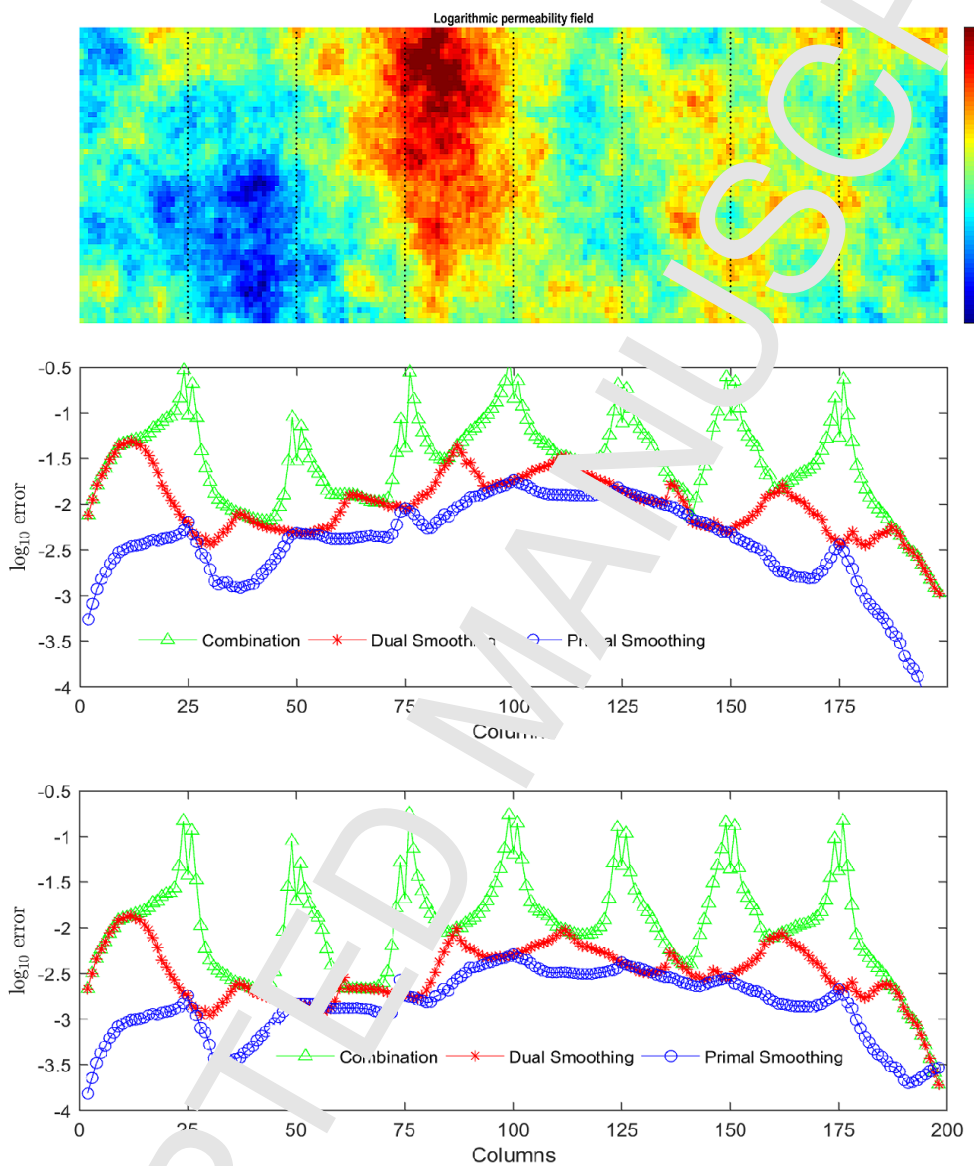

Figure 4: Example 2. A 50, 200 high contrast field with 8 primal subdomains. (1) Permeability field shown in $\log$ sca' $^{\prime}$. The biack lines are the boundaries of primal subdomains. (2) Columnwise relative error ( $3\llcorner$, f , $r$ 
(1)

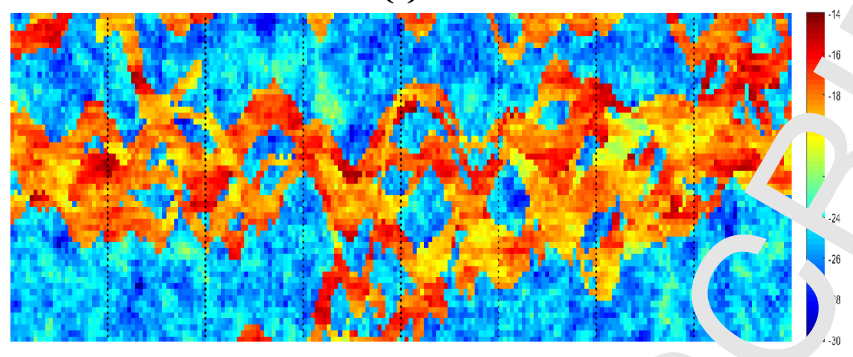

(2)

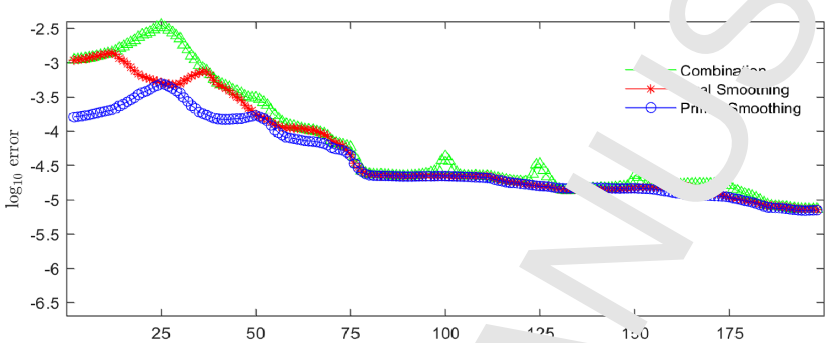

(3)

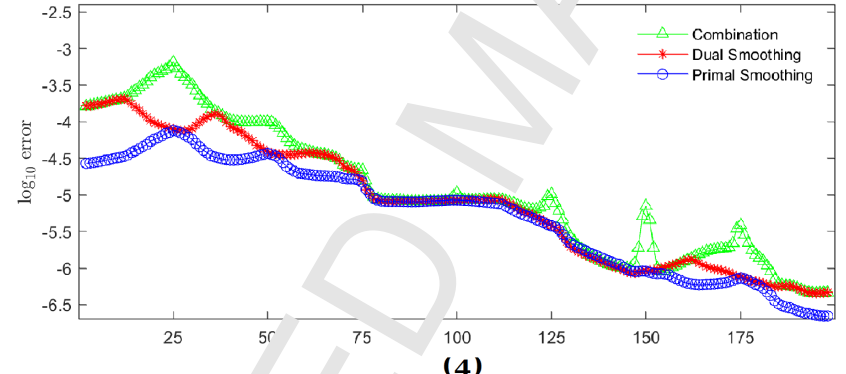

(4)

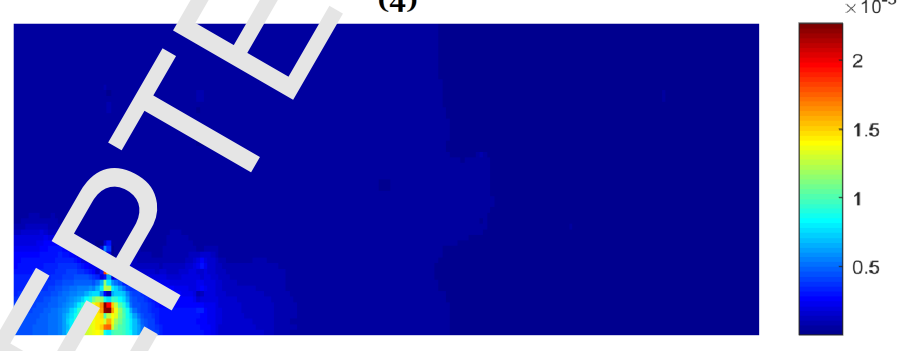

Figure 5: Example $~ A$ : $\times 200$ high contrast channelized field with 8 primal subdomains. (1) Permeability fielc shown in log scale. Black lines indicate the boundaries of primal subdomains. (2) Column-wise 1 ' 'ativ error (32) for $P$ for $m=8$. (3) Column-wise relative error (32) for $P$ for $m=4$. (4 Cell- vise relative error (31) for $P_{p s}$ for $m=4$. 
error are highlighted and concentrated close to the primal subdomain boundaries (green lines). By setting $\mathbf{u}$ as Neumann boundary conditions, the effect of dual and prima' smoothing steps can be clearly observed in 6.4 and 6.5 , respectively. Finally, the relative er 3 is shown in log scale in Figure 6.6.

\subsection{Example 5}

We consider in this study a three-dimensional example with 40 elem ${ }^{\cdots+}$ in $^{\prime}$ ach direction. The permeability field includes channelized and high contrast layers alr ng i iv' stretched cells that poses a difficult problem for numerical approximation. The values $u_{i}$ are the same in $x$ and $y$ direction, but (in average) 1000 times less permeable in $z$ dires $\lrcorner$ on, thav are plotted in Figure 7. The element size in $x$ and $y$ is the same, but it is 5 times lar rer tha in the $z$ direction, thus introducing highly stretched cells. We consider 4 subdomains wit $_{\ldots}$ - itioning in each direction separately. For example for partitioning in $x$ direction, $\mathrm{t}$ e e br u. taries of primal subdomains are layers in the $y-z$ plane, and we compute relative errol of la ers in the $y-z$ plane to see error peaks and the smoothing effect. Setting values of $P$ a Dirıchlet boundary conditions, the results are reported in Figure 8. In this Figure we con ${ }^{\circ}$ tered $r,=16$ (top) and $m=4$ (down).

\subsection{Example 6}

Finally we consider a $60 \times 200$ permeability field with . y $=10^{4} \kappa_{x}$, although this magnitude of anisotropy $\left(10^{4}\right)$ rarely occurs in reality. Consia rin , o subdomains by partitioning the domain in the $y$ direction and by taking 10 interface ndges for constructing one local solution $(m=10)$ yields the result plotted in Figure 9. We plot $3 s^{\prime}$ su amlines to visualize flow field. For such high contrast, channelized and anisotropic prc ' $. . m+t_{1_{2}}$ relative errors for $P_{p s}$ and $\mathbf{u}_{p s}$ are $4.64 E-8$ and $4.19 E-6$, respectively, in line with the 1 cults reported in Figure 9.

\section{Conclusions}

We present a proof of concept fr $:$ a ew recursive algorithm based on the combination of local finite element/volume solutions, u sbte n global accurate solutions for challenging problems, in particular in the simulation $r$ flows $1_{1}$ porous media. In all steps of this approach, i.e. solving local solutions, combining and s. nothing steps, can be handled in parallel, making the new procedure very promising $\omega_{1}$ the numerical approximation large scale problems. We tested the algorithm on different tr $>$ a $d$ three dimensional permeability fields with high contrast media. Thanks to the quality of low 1 solutions and the proposed algorithm, our method provides a reliable new approac' th t $\mathrm{c}$ c $n$ be seen as a multiscale direct solver.

\section{Acknow edgn ents}

FP wishes to unank Prof. Gustavo Buscaglia (ICMC/USP), Rafael Guiraldello (ICMC/USP) and Paola Fe raz (In ECC/UNICMAP) for some discussions about the method presented here. $\mathrm{FP}$ is grateful $\mathrm{MC} / \mathrm{USP}$ in Brazil for the hospitality during the summer of 2018, when this manu $\sim_{1}$, ... as completed. HA and APEK have been financially supported by The Danish 


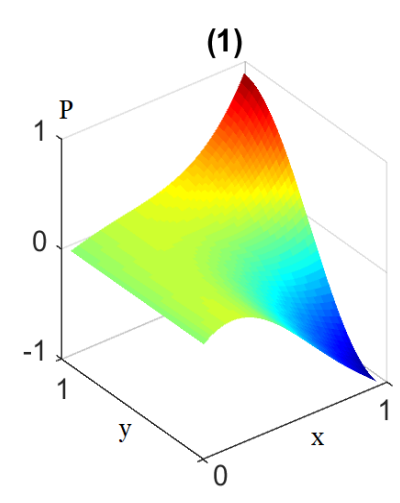

(3)

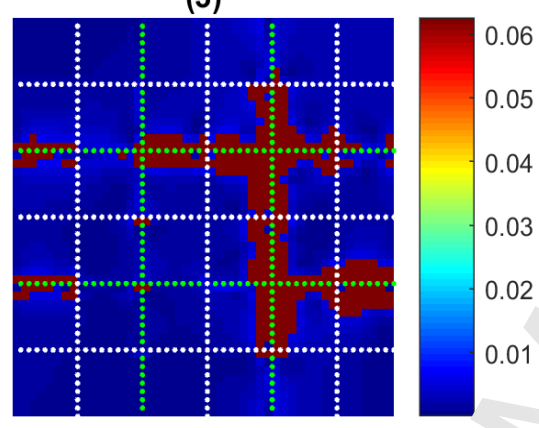

(5)
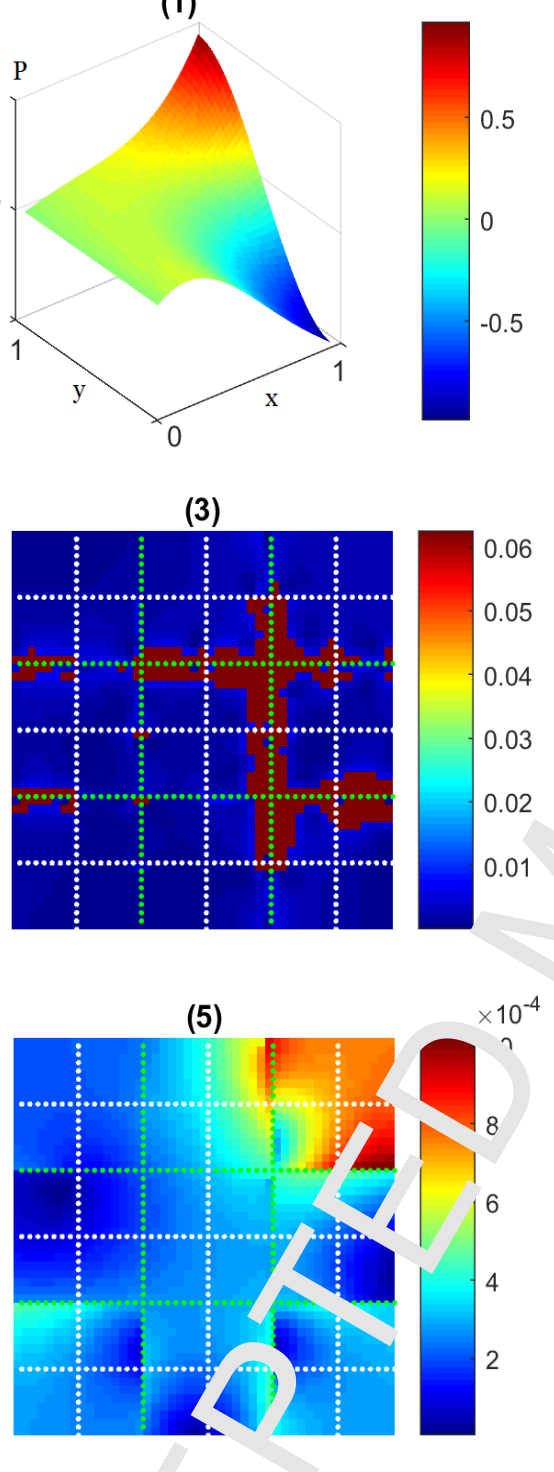
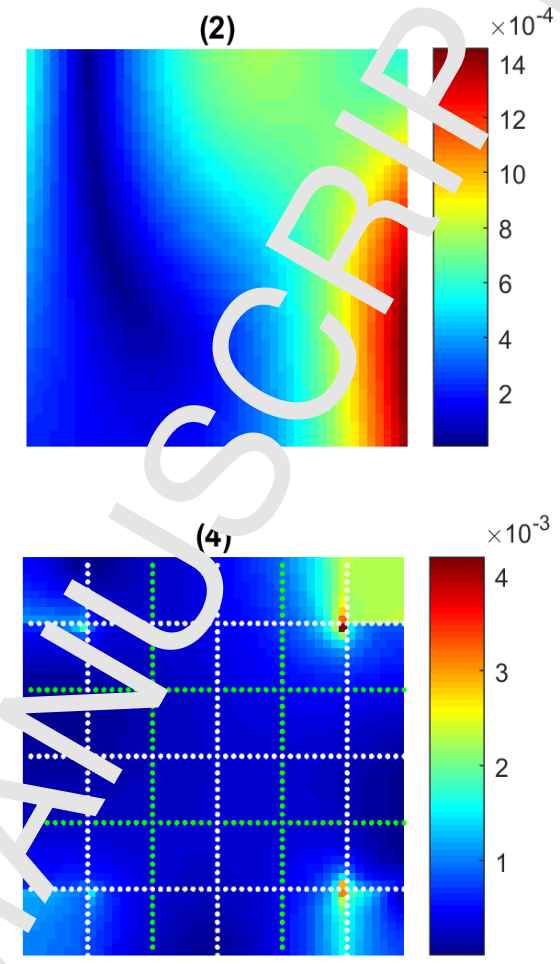

(6)

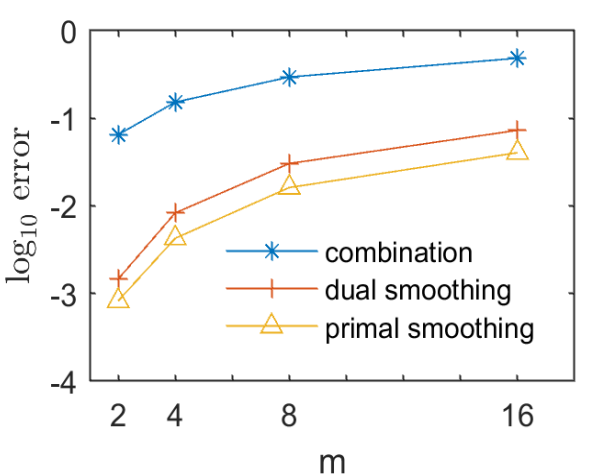

Figure 6: Examp' $\lrcorner 4$. The field $\kappa$ along with $\mu$ are reported in (33). (1) The reference solution for $P_{h}$. (2) The oferen a solution for the magnitude of $\mathbf{u}_{h}$. (3) The error magnitude of the solution $\mathbf{u}_{c l}$. ‘ Tric effect of dual smoothing. (5) The effect of primal smoothing. (6) The relative error in $\log$, sale for $m=16,8,4,2$. 

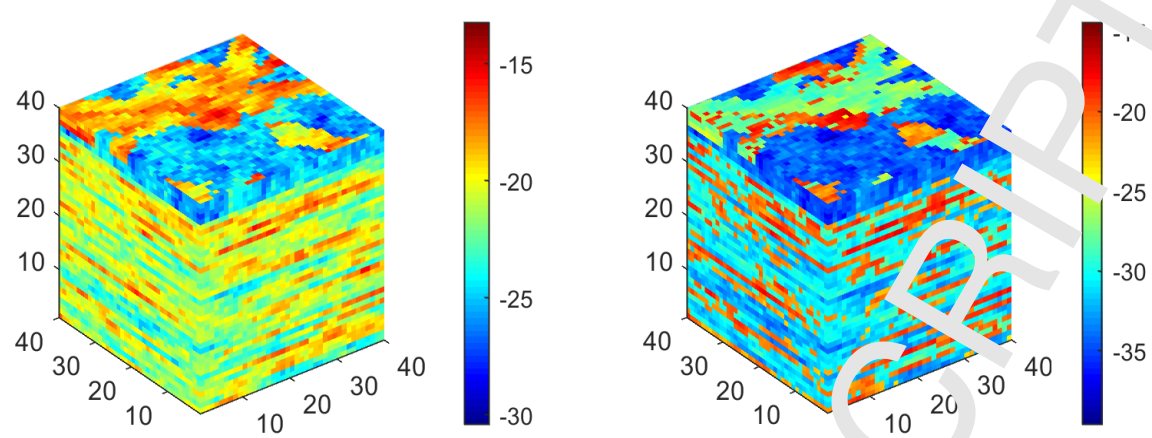

Figure 7: Example 5. Permeability field in in $\log$ scale in $\mathrm{x}$ a $\urcorner$ d $y$ dir sctions (Left) and z direction (Right).
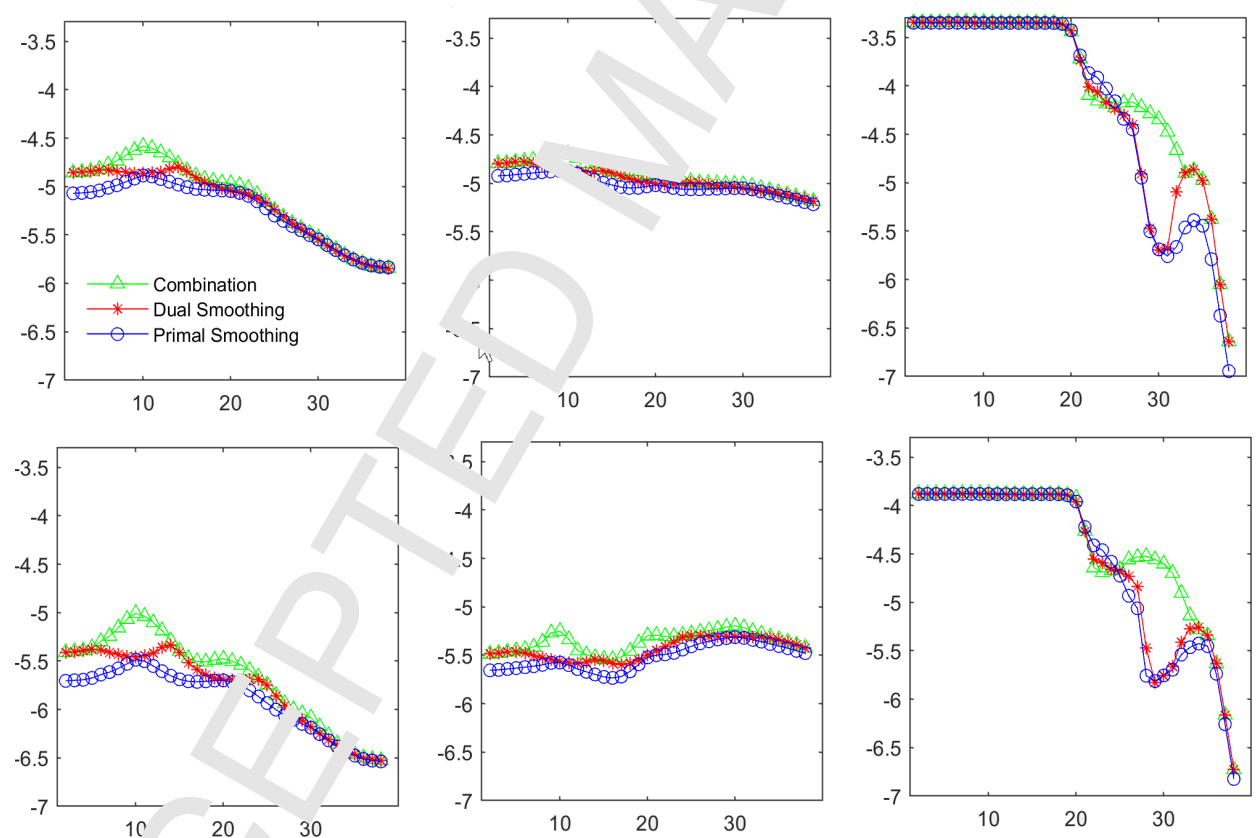

Figure 8: Examnle - relative error in log scale with partitioning in $\mathrm{x}$ (left), $\mathrm{y}$ (middle) and $\mathrm{z}$ (right) direct ons fo. $m=16$ (top) and $m=4$ (down). In all figures the vertical axis is $\log _{10}$ of the relative ei 'or of he layer in the horizontal axis. The legends are set accordingly. 


\section{ACCEPTED MANUSCRIPT}
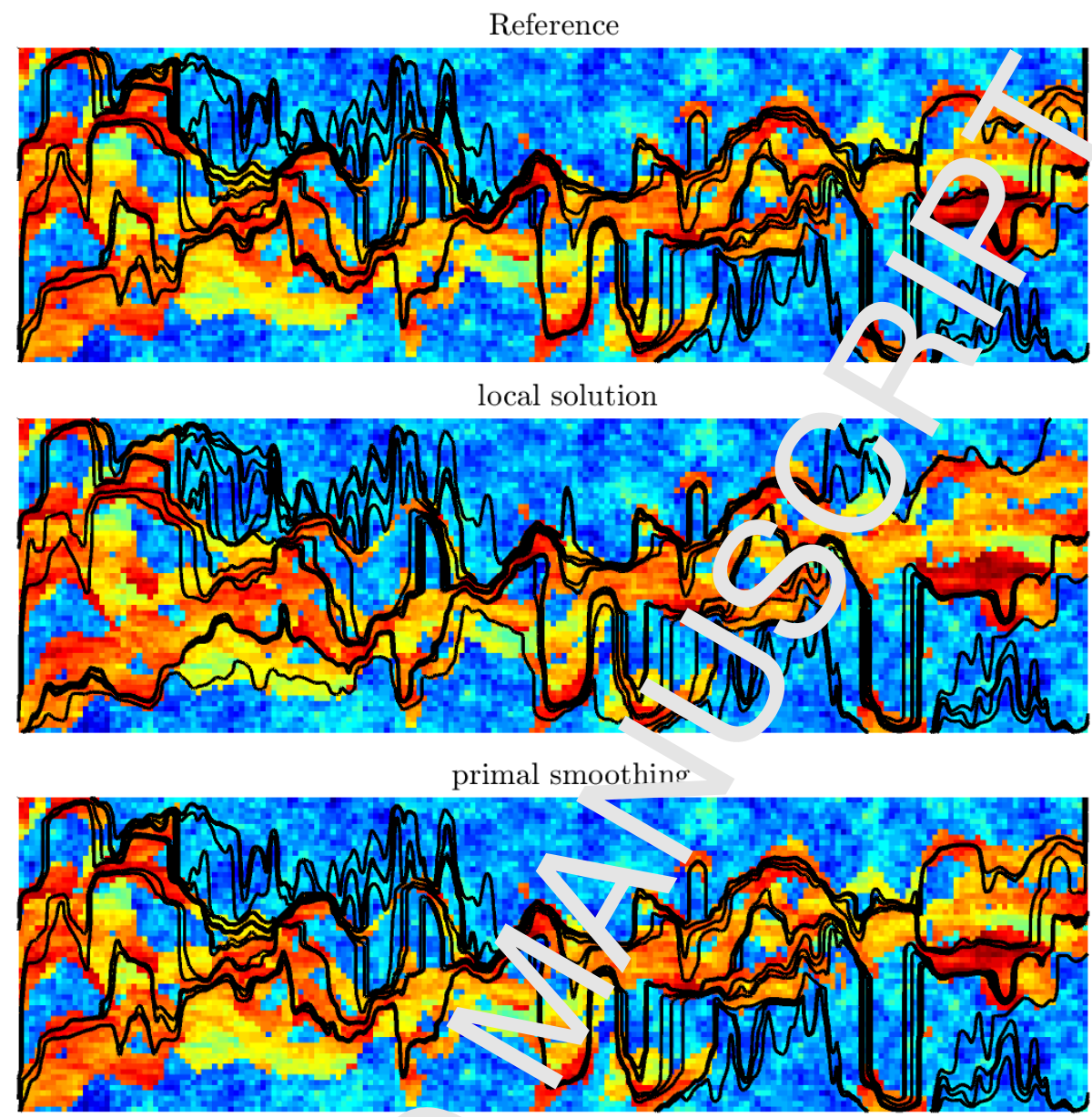

Figure 9: Example 6. The field $\kappa_{x}$ in $\log$ cale displayed along with 30 streamlines to show the flow field for the reference solut. $\cap \eta \mathrm{cop}^{\prime}, \mathbf{u}_{l s}$ (middle) and $\mathbf{u}_{p s}$ (down). $\kappa_{y}=10^{4} \kappa_{x}$.

Advanced Technology Foundation : $\eta$ the OPTION Project (J. nr 6320133). FP was funded in part by NSF-DMS $15148 \%$ s, . Science Without Borders/CNPq-Brazil grant and UT Dallas.

\section{References}

[1] L.J. Durlofsky, V Ett. ${ }^{~}:{ }_{\lrcorner} v$, V. Ginting, An adaptive local-global multi-scale finite volume element method for tv 3-phas. flow simulations, Advances in Water Resources, Vol. 30(3), (2007), 576-588.

[2] Y. Efendiev V. ' :nt $^{\prime} . \mathrm{g}, \mathrm{T}$. Hou, R. Ewing, Accurate multiscale finite element methods for two-phase flow sime ations, Journal of Computational Physics, 220(1), (2006), 155-174

[3] A. P. Engs $\mathrm{K}$ - up, Analysis of efficient preconditioned defect correction methods for nonlinear water wo Int. J. Numer. Meth. Fluids, vol 74, pages 749-773, (2014) 
[4] T. Arbogast, G. Pencheva, M.F. Wheeler, I. Yotov, A multiscale mortar mixed finite element method, SIAM Multiscale Model. Simul. 6(1) (2007) 319-346.

[5] H. Akbari, A.P. Engsig-Karup, Screening wells by multi-scale grids for mult' staø Markov Chain Monte Carlo simulation, Mathematics and Computers in Simulation 151 (201 \&) 15-2૪.

[6] E. T. Chung, Y. Efendiev, W. T. Leung, M. Vasilyeva, Y. Wang, Non-loca, nu' ,1-continua upscaling

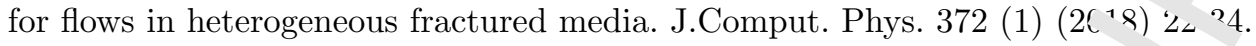

[7] J. Douglas, P.J. Paes-Leme, J.E. Roberts, J.P. Wang, A parallel iterat ve 1 rocedure applicable to the approximate solution of second order partial differential equations b. mi ad tıite element methods, Numer. Math. 65 (1) (1993) 95-108.

[8] Efendiev Y., Galvis J., Wu J. (2011) Multiscale finite element : 1ethods for high-contrast problems using local spectral basis functions. J. Comput. Phys. 230(4):93' 755

[9] R.T. Guiraldello, R.F. Ausas, F.S. Sousa, F. Pereira, G.C. I use glle The multiscale Robin coupled method for flows in porous media, Journal of Computatinnaı Phys; is 355 (2018) 1-21.

[10] A. Francisco,V. Ginting, F. Pereira, J. Rigelo, Design and in. lementation of a multiscale mixed method based on a non overlapping domain decombos. (2014) 125-138.

[11] V. Kippe, J. E. Aarnes, K. A. Lie, A Comparison of Multiscale Methods for Elliptic Problems in Porous Media Flow, Comput. Geosci. 12 (2008) 377-3s ?

[12] S. Krogstad, K. A. Lie, H. M. Nilsen, J. R. Natvı ' B. Skaflestad, E. J. Aarnes, A Multiscale Mixed Finite Element Solver for Three Phase Blac. רil F. w. SPE-118993-MS (2009).

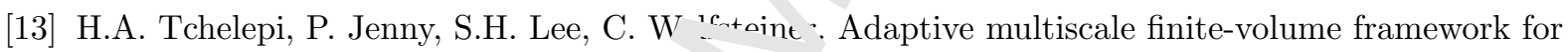
reservoir simulation. Society of Petroleum $\perp$ ' oineers, 2007, doi:10.2118/93395-PA

[14] M. Paz, W. Leigh (2001) Static Co un -ation and Substructuring. In: Integrated Matrix Analysis of Structures. Springer, Boston, $\mathrm{N}^{\mathrm{A}} \mathrm{A}$

[15] M.A. Christie, M.J. Blunt, Te ih SPL omparative solution project: A comparison of upscaling techniques, SPE Reserv. Eval Enr., 4 '2001).

[16] C. Harder, D. Paredes, F. ' alentin, .. family of multiscale hybrid-mixed finite element methods for the Darcy equation with jus coefficients, J. Comput. Phys. 245 (2013) 107-130.

[17] R. Araya, C. Harder, T, t redes, F. Valentin, Multiscale hybrid-mixed method, SIAM J. Numer. Anal. 51(6) (2013) 35 r-3r 31.

[18] R.C.C. Silva, A.F J. I sula, Local residual error estimator and adaptive finite element analysis of Poisson problems, $\mathrm{C}_{\mathrm{C}} \mathrm{npu}{ }^{+}$. Struct. 80 (2002) 2027-2034.

[19] P. Jenny, S.H цee, H A. Tchelepi, Multi-scale finite-volume method for elliptic problems in subsurface flow simı ation,. Comput. Phys. 187 (2003) 47-67.

[20] Y. Wang, 1. Haịibeygi, H. A. Tchelepi, Algebraic multi-scale solver for flow in heterogeneous porous media, Jı urnal oi Computational Physics, Vol 259, (2014), 284-303.

[21] Q. Zł^no. Z. Huang, J. Yao, Y. Wang, Y. Li, A multiscale mixed finite element method with oversa. $\mathrm{pl}^{\mathrm{i}} \mathrm{.g}$ ior modeling flow in fractured reservoirs using discrete fracture model. Journal of Computi ional and Applied Mathematics, 323 (2017) 95-110. 
[22] H. Zhou, H.A. Tchelepi, Two-stage algebraic multi-scale linear solver for highly heterogeneous reservoir models, SPE J., SPE-141473-PA (2012).

[23] K. A. Lie, O. Moyner, J. Natvig, A. Kozlova, K. Bratvedt, S. Watanabe, Z. I 1, Dᄂ 'essful application of multiscale methods in a real reservoir simulator environment, Com ${ }^{-\cdots+}$ ational Geosciences. $10.1007 / \mathrm{s} 10596-017-9627-2$. (2017)

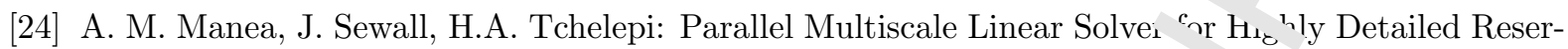
voir Models, SPE, (2015).

[25] T. Hou, X. H. Wu, A Multiscale Finite Element Method for Elliptic F • nbl ms in Composite Materials and Porous Media, J. Comput. Phys., 134 (1997), pp. 169-189.

[26] I. Lunati, S.H. Lee, An Operator Formulation of the Multiscal Finite- Volume Method with Correction Fucntion, Multiscale Model Simul., Vol. 8, No. 1, pp. 98-^ १ S'AM (2009).

[27] O. Moyner, K.A. Lie, The Multiscale Finite-Volume Methc l o' Str tigraphic Grid, SPE J. (2014).

[28] M. L. C. Christensen, K. Eskildsen, A.P. Engsig-Karup, anc M. Wakefield, Nonlinear Multigrid for Reservoir Simulation. In Society of Petroleum Enginerrs, SPE ournal, November 2015. 